\title{
New Design of Orthogonal Filter Banks Using the Cayley Transform
}

\author{
Jianping Zhou ${ }^{\dagger}$, Minh N. Do ${ }^{\dagger}$ and Jelena Kovačeviç $\$$ \\ $\dagger$ Department of Electrical and Computer Engineering \\ University of Illinois at Urabana-Champaign, Urbana, IL 61801, USA \\ $\S$ Department of Biomedical Engineering \\ Carnegie Mellon University, Pittsburgh, PA, USA
}

\begin{abstract}
It is a challenging task to design orthogonal filter banks, especially multidimensional (MD) ones. In the onedimensional (1D) two-channel finite impulse response (FIR) filter bank case, several design methods exist. Among them, designs based on spectral factorizations (by Smith and Barnwell) and designs based on lattice factorizations (by Vaidynanathan and Hoang) are the most effective and widely used. The 1D two-channel infinite impulse response (IIR) filter banks and associated wavelets were considered by Herley and Vetterli. All of these design methods are based on spectral factorization. Since in multiple dimensions, there is no factorization theorem, traditional 1D design methods fail to generalize. Tensor products can be used to construct MD orthogonal filter banks from 1D orthogonal filter banks, yielding separable filter banks. In contrast to separable filter banks, nonseparable filter banks are designed directly, and result in more freedom and better frequency selectivity. In the FIR case, Kovačević and Vetterli designed specific two-dimensional and three-dimensional nonseparable FIR orthogonal filter banks. In the IIR case, there are few design results (if any) for MD orthogonal IIR filter banks.

To design orthogonal filter banks, we must design paraunitary matrices, which leads to solving sets of nonlinear equations. The Cayley transform establishes a one-to-one mapping between paraunitary matrices and para-skewHermitian matrices. In contrast to nonlinear equations, the para-skew-Hermitian condition amounts to linear constraints on the matrix entries which are much easier to solve. We present the complete characterization of both paraunitary FIR matrices and paraunitary IIR matrices in the Cayley domain. We also propose efficient design methods for MD orthogonal filter banks and corresponding methods to impose the vanishing-moment condition.
\end{abstract}

Keywords: O rthogonal Filter Banks, Cayley Transform, Nonseparable Multidimensional Filter Design, Paraunitary Matrix.

\section{INTRODUCTION}

Over the last decade, the theory and applications of filter banks have grown rapidly [1], [2], [3], [4], [5], [6]. Among them, orthogonal filter banks received attention due to their useful properties [7], [8], [9]. First, orthogonality implies energy preservation, which guarantees that the energy of errors generated by transmission or quantization will not be amplified. Second, under certain conditions, orthogonal filter banks can be used to construct orthonormal wavelet bases [10]. The vanishing-moment condition plays a crucial role in the construction of orthonormal wavelet bases from orthogonal filter banks. An orthogonal lowpass filter is said to satisfy the $N$-th order vanishing-moment condition if it has an $N$-th order zero at aliasing frequencies.

There are two types of orthogonal filter banks: finite impulse response (FIR) filter banks and infinite impulse response (IIR) filter banks. FIR filters are more efficient to implement and hence more popular. In the $1 \mathrm{D}$ two-channel filter bank case, there exist several filter design methods. Among them, designs based on spectral

Further author information: (Send correspondence to Jianping Zhou) Jianping Zhou: E-mail: jzhou2@uiuc.edu

Minh Do: E-mail: minhdo@uiuc.edu

Jelena Kovačević: E-mail: j.kovacevic@ieee.org 
factorizations [11] and designs based on lattice factorizations [12] are the most effective and widely used. The first method, which was proposed by Smith and Barnwell [11], designs the autocorrelation sequence of a filter and then obtains that filter via spectral factorization. This method was used by Daubechies to construct celebrated family of orthogonal compactly supported wavelets [10]. However, as the size of the filter grows, spectral factorization becomes numerically ill-conditioned. Moreover, due to the lack of an MD factorization theorem, this method cannot be extended to higher dimensions. The second method, which was proposed by Vaidynanathan and Hoang [12], formulates the filter design problem as that of a polyphase transform matrix $\mathbf{U}(\boldsymbol{z})$ which has to be a paraunitary* FIR matrix, i.e.

$$
\mathbf{U}(\boldsymbol{z}) \mathbf{U}^{T}\left(\boldsymbol{z}^{-1}\right)=\mathbf{I}, \quad \text { for real coefficients. }
$$

These authors provided a complete characterization of paraunitary matrices for 1D filter banks via a lattice factorization. However, it is difficult to impose the vanishing-moment condition in a lattice factorization. As for spectral factorization methods, the lattice factorization design method cannot be extended to higher dimensions.

Although more complex, orthogonal IIR filter banks have greater design freedom, compared to FIR case. Moreover, IIR filter banks generally offer better frequency selectivity. The 1D two-channel orthogonal IIR filter banks and associated wavelets were considered by Herley and Vetterli [13]. The design method in [13] also needs spectral factorization and cannot be extended to higher dimensions.

In multiple dimensions, there are two types of orthogonal filter banks: separable and nonseparable orthogonal filter banks. For a separable filter bank, its transfer functions are products of multiple 1D orthogonal filters. Therefore, tensor products can be used to construct separable orthogonal filter banks from 1D orthogonal filter banks. In contrast to separable filter banks, nonseparable orthogonal filter banks are designed based on the MD structure directly, resulting in more freedom and better frequency selectivity. Therefore, nonseparable orthogonal filter banks have received more interest in recent years. However, due to complexity, it is a challenging task to design nonseparable orthogonal FIR and IIR filter banks. In the FIR case, Kovačević and Vetterli successfully designed specific two-dimensional (2D) and three-dimensional (3D) nonseparable orthogonal FIR filter banks [9]. However, their method could not give complete solutions and it is difficult to apply to filters with larger size. In the IIR case, there is no existing literature addressing the design problem.

In this work, we propose a novel design method for both orthogonal FIR filter banks and orthogonal IIR filter banks using the Cayley transform (CT) [14]. Designing orthogonal filter banks amounts to designing paraunitary matrices. The main feature of the $\mathrm{CT}$ is that it maps a paraunitary matrix $\mathbf{U}(\boldsymbol{z})$ to a para-skew-Hermitian ${ }^{\dagger}$ (PSH) matrix $\mathbf{H}(\boldsymbol{z})$ that satisfies

$$
\mathbf{H}\left(\boldsymbol{z}^{-1}\right)=-\mathbf{H}^{T}(\boldsymbol{z}) \text {. }
$$

Conversely, the CT maps a PSH matrix to a paraunitary matrix. Therefore, the CT establishes a one-to-one mapping between a nonlinear Stiefel manifold of paraunitary matrices and the linear space of PSH matrices, as shown in Fig. 1. Our key observation is that in contrast to solving for the nonlinear paraunitary condition in (1), the PSH condition amounts to linear constraints on the matrix entries in (2), leading to an easier design problem. The basic idea is that we first design a PSH matrix and then map it back to a paraunitary matrix by the CT. However, there are two challenges in this design approach. The first is how to impose the vanishing-moment condition in the Cayley domain. Since the vanishing-moment condition requires that the lowpass filter has a zero of specific order at aliasing frequencies, it is difficult to impose the vanishing-moment condition on the polyphase transform matrix and its CT. The second challenge is that the CT is a nonlinear operator and it destroys the FIR property, that is, the CT of an FIR matrix is no longer FIR. Thus, the CT maps a paraunitary FIR matrix to a PSH IIR matrix. We need to find a complete characterization of these PSH matrices such that their inverse CTs are FIR. In this paper, we address these issues, which lead us to a novel design method for MD orthogonal filter banks and wavelets.

${ }^{*}$ A paraunitary matrix is an extension of a unitary matrix when the matrix entries are polynomials. Paraunitary matrices are unitary on the unit circle.

${ }^{\dagger}$ A para-skew-Hermitian matrix is an extension of a skew-Hermitian matrix when the matrix entries are polynomials. Para-skew-Hermitian matrices are skew-Hermitian on the unit circle. 


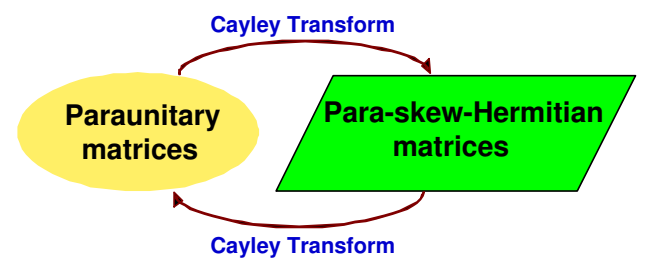

Figure 1. One to one mapping between paraunitary matrices and para-skew-Hermitian matrices via the Cayley transform.

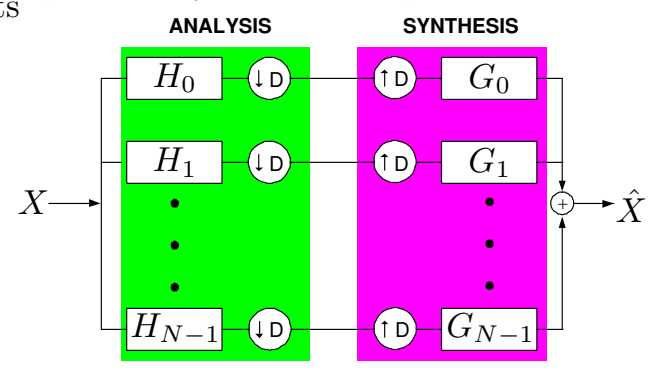

Figure 2. An MD N-channel filter bank.

The paper is organized as follows: In Section 2, we give a brief introduction to orthogonal filter banks and the Cayley transform. We introduce the special paraunitary matrix and its property in Section 3. The theory and design of orthogonal FIR filter banks, including the general multiple-channel case and the two-channel case are given in Section 4 and Section 5, respectively. The theory and design of orthogonal IIR filter banks is given in Section 6. We conclude in Section 7. Due to the space limitation, we only present the results in this paper. Please refer to [15] for proofs and design examples.

\section{ORTHOGONAL FILTER BANKS AND THE CAYLEY TRANSFORM}

We start with notation. In MD, $\boldsymbol{z}$ stands for an $M$-dimensional variable $\boldsymbol{z}=\left[z_{1}, z_{2}, \ldots, z_{M}\right]^{T}$. For an $M$ dimensional integer $\boldsymbol{k}=\left[k_{1}, k_{2}, \ldots, k_{M}\right]^{T}$, an MD power is written as $\boldsymbol{z}^{\boldsymbol{k}}=\left[z_{1}^{k_{1}}, z_{2}^{k_{2}}, \ldots, z_{M}^{k_{M}}\right]^{T}$. Specifically, $\boldsymbol{z}^{-1}$ stands for $\left[z_{1}^{-1}, z_{2}^{-1}, \ldots, z_{M}^{-1}\right]^{T}$. We will refer to a Laurent polynomial matrix as FIR or FIR matrix if all of its entries are finite length Laurent polynomials. Otherwise, it is an IIR matrix.

Consider an MD $N$-channel filter bank as shown in Fig. 2. In the filter bank, the input $X(\boldsymbol{z})$ is fed to the $N$ subband filters, $H_{0}(\boldsymbol{z})$ to $H_{N-1}(\boldsymbol{z})$. After filtering, each output is downsampled by an $M \times M$ sampling matrix $D$ with the sampling rate $|\operatorname{det} D|$. The $N$ branch outputs of the analysis part are upsampled with the same sampling matrix and subsequently filtered by a bank of subband filters, $G_{0}(\boldsymbol{z})$ to $G_{N-1}(\boldsymbol{z})$. Finally, all the $N$ branches are added together to generate a single output $\hat{X}(\boldsymbol{z})$. The whole system can be divided into two parts, analysis part and synthesis part. The analysis part consists of subband filtering and downsampling, while the synthesis part consists of upsampling and subband filtering. In the analysis and design of filter banks, polyphase representation is often used as it allows for time-invariant analysis in the polyphase domain. In the polyphase domain, the analysis part and synthesis part can be represented by $N \times N$ matrices $\mathbf{H}_{p}(\boldsymbol{z})$ and $\mathbf{G}_{p}(\boldsymbol{z})$ respectively. We are interested in the critically sampled filter bank in which the sampling rate is equal to the number of channels, i.e., $|\operatorname{det} D|=N$. Also, perfect reconstruction is a desirable property of a system so that the output $\hat{X}(\boldsymbol{z})$ is always equal to the input $X(\boldsymbol{z})$. Under these assumptions, the perfect reconstruction condition is equivalent to $\mathbf{H}_{p}(\boldsymbol{z}) \mathbf{G}_{p}(\boldsymbol{z})=\mathbf{I}$. For orthogonal filter banks, $\mathbf{G}_{p}(\boldsymbol{z})=\mathbf{H}_{p}^{T}\left(\boldsymbol{z}^{-\mathbf{1}}\right)$, and thus $\mathbf{H}_{p}(\boldsymbol{z})$ is a paraunitary matrix. Throughout the paper, we will always refer to $M$ as the number of dimensions; $N$ as the number of channels.

The complex nonlinear paraunitary constraint leads to a difficult nonlinear spectral factorization problem. The Cayley transform is a powerful tool to convert a nonlinear problem into a linear one. It is widely used in control theory and Lie groups [14]. The Cayley transform of a matrix $\mathbf{U}(\boldsymbol{z})$ is defined as

$$
\mathbf{H}(\boldsymbol{z})=(\mathbf{I}+\mathbf{U}(\boldsymbol{z}))^{-1}(\mathbf{I}-\mathbf{U}(\boldsymbol{z})) .
$$


The inverse of the $\mathrm{CT}$ is

$$
\mathbf{U}(\boldsymbol{z})=(\mathbf{I}+\mathbf{H}(\boldsymbol{z}))^{-1}(\mathbf{I}-\mathbf{H}(\boldsymbol{z})) .
$$

By definition, the $\mathrm{CT}$ is formulated as the product of two matrices. Actually, it can also be expressed in an alternative way:

Proposition 1. Suppose that $\mathbf{U}(\boldsymbol{z})$ is a matrix and $\mathbf{H}(\boldsymbol{z})$ is its $C T$. Then

$$
\mathbf{H}(\boldsymbol{z})=2(\mathbf{I}+\mathbf{U}(\boldsymbol{z}))^{-1}-\mathbf{I} \quad \text { and } \quad \mathbf{U}(\boldsymbol{z})=2(\mathbf{I}+\mathbf{H}(\boldsymbol{z}))^{-1}-\mathbf{I} .
$$

The Cayley transform is a matrix generalization of the bilinear transform [16](pp. 415-417), which is defined as $s=(1+z)^{-1}(1+z)$. The bilinear transform maps the imaginary axis of the complex $s$-plane onto the unit circle in the complex $z$-plane. It is widely used in signal processing theory, for example, to map continuous-time systems to discrete-time systems. Similarly to the bilinear transform, the Cayley transform maps paraunitary matrices to PSH matrces.

Proposition 2. The Cayley transform of a paraunitary matrix is a para-skew-Hermitian matrix. Conversely, the Cayley transform of a para-skew-Hermitian matrix is a paraunitary matrix.

Based on Proposition 2, the CT establishes a one-to-one mapping between paraunitary matrices and PSH matrices. It maps the nonlinear Stiefel manifold of paraunitary matrices to the linear space of PSH matrices. In contrast to solving for the nonlinear paraunitary condition, the PSH condition amounts to linear constraints on the matrix entries.

To illustrate this, consider the two-channel case. Let $\mathbf{U}(\boldsymbol{z})$ be a $2 \times 2$ paraunitary matrix with

$$
\mathbf{U}(\boldsymbol{z})=\left(\begin{array}{cc}
U_{00}(\boldsymbol{z}) & U_{01}(\boldsymbol{z}) \\
U_{10}(\boldsymbol{z}) & U_{11}(\boldsymbol{z})
\end{array}\right)
$$

then it satisfies $\mathbf{U}(\boldsymbol{z}) \mathbf{U}^{T}\left(\boldsymbol{z}^{-1}\right)=\mathbf{I}$. Specifically,

$$
\begin{aligned}
& U_{00}(\boldsymbol{z}) U_{00}\left(\boldsymbol{z}^{-1}\right)+U_{01}(\boldsymbol{z}) U_{01}\left(\boldsymbol{z}^{-1}\right)=1, \\
& U_{00}(\boldsymbol{z}) U_{10}\left(\boldsymbol{z}^{-1}\right)+U_{01}(\boldsymbol{z}) U_{11}\left(\boldsymbol{z}^{-1}\right)=0, \\
& U_{10}(\boldsymbol{z}) U_{10}\left(\boldsymbol{z}^{-1}\right)+U_{11}(\boldsymbol{z}) U_{11}\left(\boldsymbol{z}^{-1}\right)=1 .
\end{aligned}
$$

Solving the system involves solving three nonlinear equations with respect to coefficients of $\mathbf{U}(\boldsymbol{z})$. In contrast, let $\mathbf{H}(\boldsymbol{z})$ be a $2 \times 2$ PSH matrix with

$$
\mathbf{H}(\boldsymbol{z})=\left(\begin{array}{cc}
H_{00}(\boldsymbol{z}) & H_{01}(\boldsymbol{z}) \\
H_{10}(\boldsymbol{z}) & H_{11}(\boldsymbol{z})
\end{array}\right)
$$

then it satisfies $\mathbf{H}\left(\boldsymbol{z}^{-1}\right)=-\mathbf{H}^{T}(\boldsymbol{z})$. Specifically,

$$
H_{00}\left(\boldsymbol{z}^{-1}\right)=-H_{00}(\boldsymbol{z}), \quad H_{11}\left(\boldsymbol{z}^{-1}\right)=-H_{11}(\boldsymbol{z}), \quad \text { and } \quad H_{01}\left(\boldsymbol{z}^{-1}\right)=-H_{10}(\boldsymbol{z}) .
$$

Here, solving the system involves solving only three independent linear equations with respect to coefficients of $\mathbf{H}(\boldsymbol{z})$. Moreover, we do not need to solve these three equations in the design. Instead, we only need to design two anti-symmetric linear phase filters independently, $H_{00}(\boldsymbol{z})$ and $H_{11}(\boldsymbol{z})$, choose one arbitrary filter $H_{10}(\boldsymbol{z})$ leading to $H_{01}(\boldsymbol{z})$ as in (6). 


\section{THE SPECIAL PARAUNITARY MATRIX}

Using the CT, the design problem of paraunitary matrices is simplified to that of PSH matrices. However, it still involves too many equations, especially when the number of channels is large. The special paraunitary matrix is prosperous to simplify the design.

Special paraunitary matrices are a special class of paraunitary matrices.

Definition. 1. A paraunitary matrix $\mathbf{U}(\boldsymbol{z})$ is said to be a special paraunitary (SP) matrix $\ddagger$ if its determinant equals to 1.

SP matrices satisfy all the property of paraunitary matrices. The product of two SP matrices is also SP. Therefore, the set of SP matrices is also a Stiefel manifold. Moreover, it is easier to design SP matrices than paraunitary matrices.

Proposition 3. Suppose $\mathbf{U}(\boldsymbol{z})$ is a matrix and its determinant equals to 1 . Then $\mathbf{U}(\boldsymbol{z})$ is $S P$ if and only if

$$
U_{i, j}\left(\boldsymbol{z}^{-1}\right)=\operatorname{minor} U_{i, j}(\boldsymbol{z}),
$$

where $U_{i, j}(\boldsymbol{z})$ is the entry of $\mathbf{U}(\boldsymbol{z})$ at $(i, j)$.

By Propostion 3, the design of SP matrix amounts to the determinant condition and (7). By (7), we can get rid of some nonlinear equations. To illustrate this, consider the two-channel case. Suppose $\mathbf{U}(\boldsymbol{z})$ is a $2 \times 2 \mathrm{SP}$ matrix, then (7) becomes

$$
U_{10}(\boldsymbol{z})=-U_{01}\left(\boldsymbol{z}^{-1}\right) \quad \text { and } \quad U_{11}(\boldsymbol{z})=U_{00}\left(\boldsymbol{z}^{-1}\right),
$$

and the determinant condition becomes:

$$
U_{00}(\boldsymbol{z}) U_{00}\left(\boldsymbol{z}^{-1}\right)+U_{01}(\boldsymbol{z}) U_{01}\left(\boldsymbol{z}^{-1}\right)=1 .
$$

Therefore the complete characterization of $\mathbf{U}(\boldsymbol{z})$ is

$$
\mathbf{U}(\boldsymbol{z})=\left(\begin{array}{cc}
U_{00}(\boldsymbol{z}) & U_{01}(\boldsymbol{z}) \\
-U_{01}\left(\boldsymbol{z}^{-1}\right) & U_{00}\left(\boldsymbol{z}^{-1}\right)
\end{array}\right),
$$

where $U_{00}(\boldsymbol{z})$ and $U_{01}(\boldsymbol{z})$ satisfies the power complementary property given in (8). Note that (8) is the same as (5). Therefore, for $2 \times 2 \mathrm{SP}$ matrices, we need to solve only one equation of the three equations required by the general $2 \times 2$ paraunitary matrices.

One useful property of the SP matrix is that every paraunitary matrix can be represented as the product of an SP matrix and a diagonal paraunitary matrix:

TheOrem 1. Suppose $\mathbf{U}(\boldsymbol{z})$ is a paraunitary matrix. Then $\mathbf{U}(\boldsymbol{z})$ can be written as $\mathbf{U}(\boldsymbol{z})=\mathbf{U}_{s}(\boldsymbol{z}) \mathbf{U}_{d}(\boldsymbol{z})$, where both $\mathbf{U}_{s}(\boldsymbol{z})$ and $\mathbf{U}_{d}(\boldsymbol{z})$ are paraunitary, and

$$
\operatorname{det} \mathbf{U}_{s}(\boldsymbol{z})=1 \text { and } \mathbf{U}_{d}(\boldsymbol{z})=\operatorname{diag}(1, \cdots, 1, \operatorname{det} \mathbf{U}(\boldsymbol{z})) .
$$

Moreover, if $\mathbf{U}(\boldsymbol{z})$ is FIR, then $\operatorname{det} \mathbf{U}(\boldsymbol{z})$ is a pure delay.

By Theorem 1, a paraunitary matrix can be "normalized" as an SP matrix, where the first $N-1$ columns of the matrix are kept the same and the last column is multiplied with the allpass filter $(\operatorname{det} \mathbf{U}(\boldsymbol{z}))^{-1}$. It means that the first $N-1$ filters of the corresponding orthogonal filter bank are kept the same and the last filter is changed only in the phase. In the FIR case, the change is just a pure delay. SP matrices can be used to simplify our design problem. To illustrate this, consider the two-channel case. By Theorem 1 and (9), the characterization of the $2 \times 2$ paraunitary matrix is

$$
\mathbf{U}(\boldsymbol{z})=\mathbf{U}_{s}(\boldsymbol{z})\left(\begin{array}{cc}
1 & 0 \\
0 & \Delta(\boldsymbol{z})
\end{array}\right)=\left(\begin{array}{cc}
U_{00}(\boldsymbol{z}) & U_{01}(\boldsymbol{z}) \Delta(\boldsymbol{z}) \\
-U_{01}\left(\boldsymbol{z}^{-1}\right) & U_{00}\left(\boldsymbol{z}^{-1}\right) \Delta(\boldsymbol{z})
\end{array}\right),
$$

\footnotetext{
$\ddagger$ The concept of the special paraunitary matrix is similiar to that of the special orthogonal matrix. An orthogonal matrix is said to be a special orthogonal matrix if its determinant equals to 1 .
} 


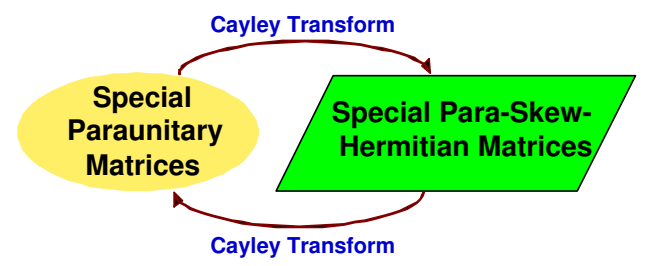

Figure 3. One-to-one mapping between SP matrices and SPSH matrices

where $U_{00}(\boldsymbol{z})$ and $U_{01}(\boldsymbol{z})$ satisfy the power complementary property. Herley and Vetterli showed the similar result for the $1 \mathrm{D}$ case in [13].

By Proposition 2, the characterization of paraunitary matrices in the Cayley domain is PSH matrices. For an SP matrix $\mathbf{U}(\boldsymbol{z}), \operatorname{det} \mathbf{U}(\boldsymbol{z})=1$. Let $\mathbf{H}(z)$ be the CT of $\mathbf{U}(\boldsymbol{z})$, then

$$
\mathbf{U}(\boldsymbol{z})=(\mathbf{I}+\mathbf{H}(z))^{-1}(\mathbf{I}-\mathbf{H}(z)) .
$$

Therefore, the necessary and sufficient condition for the CT of $\mathbf{H}(z)$ to be SP is

$$
\operatorname{det}(\mathbf{I}+\mathbf{H}(z))=\operatorname{det}(\mathbf{I}-\mathbf{H}(z)) .
$$

Accordingly, if a PSH matrix satisfies (10), it is said to be a special PSH (SPSH) matrix. Thus, the characterization of SP matrices in the Cayley domain is SPSH matrices.

For the two-channel case, the characterization is simple.

Proposition 4. Suppose $\mathbf{H}(z)$ is a $2 \times 2$ PSH matrix. Then it is SPSH if and only if its trace equals to 0.

It is clear that the set of $2 \times 2 \mathrm{SPSH}$ matrices is also a linear space. Therefore, the CT maps the complicated nonlinear Stiefel manifold of $2 \times 2 \mathrm{SP}$ matrices to the linear space of $2 \times 2 \mathrm{SPSH}$ matrices as shown in Fig. 3 .

\section{ORTHOGONAL FIR FILTER BANKS}

To design orthogonal FIR filter banks, we need to design paraunitary FIR matrices. By Theorem 1, any paraunitary FIR matrix can be "normalized" as an SP FIR matrix and the only difference between two corresponding filter banks is a pure delay in the last highpass filter. Therefore, the design problem of paraunitary FIR matrices is equivalent to that of SP FIR matrices. In this section, unless explicitly stated otherwise, assume that $\mathbf{U}(\boldsymbol{z})$ is an $N \times N$ SP FIR matrix, and $\mathbf{H}(\boldsymbol{z})$ is its CT.

As mentioned in Section 1, the FIR property is destroyed by the CT because of the matrix inversion operation in (3). Specifically, $\mathbf{U}(\boldsymbol{z})$ is an FIR matrix, but $(\mathbf{I}+\mathbf{U}(\boldsymbol{z}))^{-1}$ is in general not an FIR matrix, neither does $\mathbf{H}(\boldsymbol{z})$. Thus, we need to find a complete characterization of these SPSH matrices such that their CTs are FIR. The CT of $\mathbf{U}(\boldsymbol{z})$ is $\mathbf{H}(\boldsymbol{z})=(\mathbf{I}+\mathbf{U}(\boldsymbol{z}))^{-1}(\mathbf{I}-\mathbf{U}(\boldsymbol{z}))$, where $(\mathbf{I}+\mathbf{U}(\boldsymbol{z}))^{-1}$ can be IIR. Note that the inverse of a nonsingular matrix $\mathbf{A}$ can be expressed as $\mathbf{A}^{-1}=(\operatorname{det} \mathbf{A})^{-1} \operatorname{adj} \mathbf{A}$, where $\operatorname{adj} \mathbf{A}$ is the adjoint of $\mathbf{A}$. Therefore,

$$
\mathbf{H}(\boldsymbol{z})=(\mathbf{I}+\mathbf{U}(\boldsymbol{z}))^{-1}(\mathbf{I}-\mathbf{U}(\boldsymbol{z}))=\frac{\operatorname{adj}(\mathbf{I}+\mathbf{U}(\boldsymbol{z}))(\mathbf{I}-\mathbf{U}(\boldsymbol{z}))}{\operatorname{det}(\mathbf{I}+\mathbf{U}(\boldsymbol{z}))} .
$$

The essential observation is that $\mathbf{H}(\boldsymbol{z})$ can be represented as the quotient of an FIR matrix, adj $(\mathbf{I}+\mathbf{U}(\boldsymbol{z}))(\mathbf{I}-$ $\mathbf{U}(\boldsymbol{z}))$, and an FIR filter, $\operatorname{det}(\mathbf{I}+\mathbf{U}(\boldsymbol{z}))$. Let $D(\boldsymbol{z})$ and $\mathbf{H}^{\prime}(\boldsymbol{z})$ be the scaled denominator and numerator respectively, i.e.,

$$
\begin{aligned}
D(\boldsymbol{z}) & \stackrel{\text { def }}{=} 2^{-N+1} \operatorname{det}(\mathbf{I}+\mathbf{U}(\boldsymbol{z})), \\
\mathbf{H}^{\prime}(\boldsymbol{z}) & \stackrel{\text { def }}{=} 2^{-N+1} \operatorname{adj}(\mathbf{I}+\mathbf{U}(\boldsymbol{z}))(\mathbf{I}-\mathbf{U}(\boldsymbol{z})),
\end{aligned}
$$


where the scalar $2^{-N+1}$ is introduced for later convenience. Then $\mathbf{H}(\boldsymbol{z})$ can be expressed as

$$
\mathbf{H}(\boldsymbol{z})=\frac{\mathbf{H}^{\prime}(\boldsymbol{z})}{D(\boldsymbol{z})} .
$$

From Proposition 1,

$$
\operatorname{det}(\mathbf{I}+\mathbf{U}(\boldsymbol{z}))=2^{N} \operatorname{det}(\mathbf{I}+\mathbf{H}(\boldsymbol{z}))^{-1} .
$$

From (11), we have an alternative expression for $D(\boldsymbol{z})$ :

$$
D(\boldsymbol{z})=2 \operatorname{det}(\mathbf{I}+\mathbf{H}(\boldsymbol{z}))^{-1} .
$$

Our task now is to obtain the characterization of both $D(\boldsymbol{z})$ and $\mathbf{H}^{\prime}(\boldsymbol{z})$.

Lemma 1. Suppose $\mathbf{U}(\boldsymbol{z})$ is an SP FIR matrix. Then the Cayley transform of $\mathbf{U}(\boldsymbol{z}), \mathbf{H}(\boldsymbol{z})$, can be written as $D(\boldsymbol{z})^{-1} \mathbf{H}^{\prime}(\boldsymbol{z})$, where $D(\boldsymbol{z})$ is a symmetric FIR filter and $\mathbf{H}^{\prime}(\boldsymbol{z})$ is a PSH FIR matrix. Moreover, if $D(\boldsymbol{z})$ and $\mathbf{H}^{\prime}(\boldsymbol{z})$ are coprime, then they are unique for each SP FIR matrix $\mathbf{U}(\boldsymbol{z})$.

According to Lemma 1, the problem of designing $\mathbf{H}(\boldsymbol{z})$ is converted into that of designing a symmetric FIR filter $D(\boldsymbol{z})$ and a PSH FIR matrix $\mathbf{H}^{\prime}(\boldsymbol{z})$. However, they are not independent of each other, as shown in the following Lemma:

Lemma 2. Suppose that $\mathbf{U}(\boldsymbol{z})$ is an SP matrix, and $D(\boldsymbol{z})$ and $\mathbf{H}^{\prime}(\boldsymbol{z})$ are given by (11) and (12), respectively. Then

$$
2 D(\boldsymbol{z})^{N-1}=\operatorname{det}\left(D(\boldsymbol{z}) \mathbf{I}+\mathbf{H}^{\prime}(\boldsymbol{z})\right) .
$$

We are now in a position to formulate the complete characterization of paraunitary FIR matrices in the Cayley domain.

TheOREm 2. The CT of a matrix $\mathbf{H}(\boldsymbol{z})$ is an SP FIR matrix if and only if it can be written as $\mathbf{H}(\boldsymbol{z})=$ $D(\boldsymbol{z})^{-1} \mathbf{H}^{\prime}(\boldsymbol{z})$, where $D(\boldsymbol{z})$ is a symmetric FIR filter and $\mathbf{H}^{\prime}(\boldsymbol{z})$ is a PSH FIR matrix, and they satisfy the following two conditions:

1. $2 D(\boldsymbol{z})^{N-1}=\operatorname{det}\left(D(\boldsymbol{z}) \mathbf{I}+\mathbf{H}^{\prime}(\boldsymbol{z})\right)$;

2. $D^{N-2}(\boldsymbol{z})$ is a common factor of all minors of $D(\boldsymbol{z}) \mathbf{I}+\mathbf{H}^{\prime}(\boldsymbol{z})$.

Moreover, the CT of $\mathbf{H}(\boldsymbol{z})$ can be written as

$$
\mathbf{U}(\boldsymbol{z})=\frac{\operatorname{adj}\left(D(\boldsymbol{z}) \mathbf{I}+\mathbf{H}^{\prime}(\boldsymbol{z})\right)}{D(\boldsymbol{z})^{N-2}}-\mathbf{I} .
$$

Therefore, our problem of designing an SP FIR matrix $\mathbf{U}(\boldsymbol{z})$ is converted to a problem of designing a symmetric FIR filter $D(\boldsymbol{z})$ and a PSH FIR matrix $\mathbf{H}^{\prime}(\boldsymbol{z})$, and they satisfy the two conditions given in Theorem 2 . The first condition guarantees that $\mathbf{U}(\boldsymbol{z})$ is SP and the second condition guarantees that $\mathbf{U}(\boldsymbol{z})$ is FIR. In the two-channel case, the second condition holds automatically. Thus, we can design $D(\boldsymbol{z})$ and $\mathbf{H}^{\prime}(\boldsymbol{z})$ first, then compute the CT of $\mathbf{H}(\boldsymbol{z})=D(\boldsymbol{z})^{-1} \mathbf{H}^{\prime}(\boldsymbol{z})$ by a simple formula without matrix inversion manipulation given in $(17)$. 


\section{TWO-CHANNEL ORTHOGONAL FIR FILTER BANKS}

Among MD orthogonal filter banks, two-channel ones are the simplest and most popular. Also, this is the case of most interest to us. In this special case, we can simplify our design method. In this section, suppose $D(\boldsymbol{z})$ is a symmetric FIR filter and $\mathbf{H}^{\prime}(\boldsymbol{z})$ is a $2 \times 2$ PSH FIR matrix, as defined in (11) and (12). The goal here is to express $\mathbf{U}(\boldsymbol{z})$ directly as an FIR matrix using the terms from $D(\boldsymbol{z})$ and $\mathbf{H}^{\prime}(\boldsymbol{z})$ so that we can impose further conditions on them (e.g., vanishing-moment condition).

The characterization of SP matrices in the Cayley domain is SPSH matrices. Therefore, $\mathbf{H}(\boldsymbol{z})$ is a $2 \times 2$ SPSH matrix. By Proposition $4, \operatorname{tr} \mathbf{H}(\boldsymbol{z})=0$. From (15), $\mathbf{H}^{\prime}(\boldsymbol{z})$ can be written as

$$
\mathbf{H}^{\prime}(\boldsymbol{z})=D(\boldsymbol{z}) \mathbf{H}(\boldsymbol{z})=\frac{2 \mathbf{H}(\boldsymbol{z})}{\operatorname{det}(\mathbf{I}+\mathbf{H}(\boldsymbol{z}))} .
$$

Therefore, $\operatorname{tr} \mathbf{H}^{\prime}(\boldsymbol{z})=0$, i.e., $\mathbf{H}^{\prime}(\boldsymbol{z})$ is also an SPSH matrix. One interesting observation is that $\mathbf{H}^{\prime}(\boldsymbol{z})$ is an SPSH FIR matrix, while $\mathbf{H}(\boldsymbol{z})$ is an SPSH (generally IIR) matrix. Therefore, (18) can be regarded as a mapping from the SPSH IIR matrix to the SPSH FIR matrix. The CT destroys the FIR property from $\mathbf{U}(\boldsymbol{z})$ to $\mathbf{H}(\boldsymbol{z})$. Here this mapping "restores" the the FIR property from $\mathbf{H}(\boldsymbol{z})$ to $\mathbf{H}^{\prime}(\boldsymbol{z})$. Put them together, we can map the SP FIR matrix to the SPSH FIR matrix! To further our discussion, a formal definition of the mapping given in (18) is necessary:

Definition. 2. The post-Cayley transform (PCT) of a rational matrix $\mathbf{H}(\boldsymbol{z})$ is defined as

$$
\mathbf{H}^{\prime}(\boldsymbol{z})=\frac{2 \mathbf{H}(\boldsymbol{z})}{\operatorname{det}(\mathbf{I}+\mathbf{H}(\boldsymbol{z}))} .
$$

Using the CT, we map the SP FIR matrix to SPSH IIR matrix. Using the PCT, we can map the SPSH IIR matrix to the SPSH FIR matrix. We called this sequence of mappings the FIR-Cayley transform (FCT). The FCT of an SP FIR matrix $\mathbf{U}(\boldsymbol{z})$ can be obtained by (3) and (14):

$$
\mathbf{H}^{\prime}(\boldsymbol{z})=\frac{2 \mathbf{H}(\boldsymbol{z})}{\operatorname{det}(\mathbf{I}+\mathbf{H}(\boldsymbol{z}))}=2^{-1} \operatorname{det}(\mathbf{I}+\mathbf{U}(\boldsymbol{z}))(\mathbf{I}+\mathbf{U}(\boldsymbol{z}))^{-1}(\mathbf{I}-\mathbf{U}(\boldsymbol{z}))=2^{-1} \operatorname{adj}(\mathbf{I}+\mathbf{U}(\boldsymbol{z}))(\mathbf{I}-\mathbf{U}(\boldsymbol{z})) .
$$

To use the FCT in our design, we need to compute its inverse mapping. For the CT, we know its inverse is itself. How about the PCT? First, is the PCT a one-to-one mapping?

Proposition 5. Suppose that $\mathbf{H}_{1}(\boldsymbol{z})$ and $\mathbf{H}_{2}(\boldsymbol{z})$ are two $2 \times 2$ rational matrices such that their PCTs are equal. Then they are equal or

$$
\mathbf{H}_{2}(\boldsymbol{z})=\frac{\mathbf{H}_{1}(\boldsymbol{z})}{\operatorname{det}\left(\mathbf{H}_{1}(\boldsymbol{z})\right)} .
$$

By Propostion 5, if the determinant of $\mathbf{H}(\boldsymbol{z})$ is 1, then the mapping is one-to-one. Otherwise, the mapping is two-to-one.

Secondly, what's the inverse transform of the PCT? By Theorem 2,

$$
2 D(\boldsymbol{z})=\operatorname{det}\left(D(\boldsymbol{z}) \mathbf{I}+\mathbf{H}^{\prime}(\boldsymbol{z})\right) .
$$

Since $\mathbf{H}^{\prime}(\boldsymbol{z})$ is SPSH,

$$
D^{2}(\boldsymbol{z})-2 D(\boldsymbol{z})+\operatorname{det} \mathbf{H}^{\prime}(\boldsymbol{z})=0 .
$$

Then we can obtain the inverse mapping of the PCT in the following proposition:

Proposition 6. Suppose $\mathbf{H}^{\prime}(\boldsymbol{z})$ is a $2 \times 2$ SPSH FIR matrix. Then the inverse PCT transform of $\mathbf{H}^{\prime}(\boldsymbol{z})$ exists if and only if $\sqrt{1-\operatorname{det}\left(\mathbf{H}^{\prime}(\boldsymbol{z})\right)}$ is FIR. If it exists, it is given by

$$
\mathbf{H}(\boldsymbol{z})=\frac{\mathbf{H}^{\prime}(\boldsymbol{z})}{1 \pm \sqrt{1-\operatorname{det}\left(\mathbf{H}^{\prime}(\boldsymbol{z})\right)}} .
$$




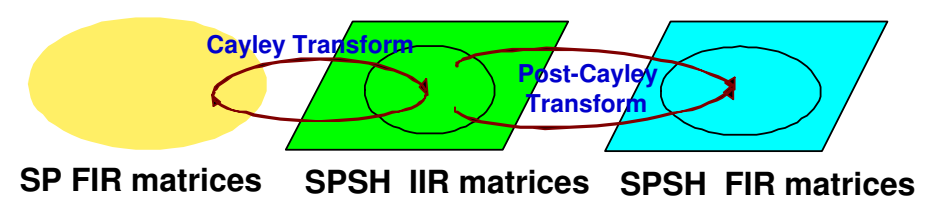

Figure 4. Mapping from SP FIR matrices to SPSH FIR matrices

By Proposition 6, the characterization of SP FIR matrices in the FCT domain is a subset of SPSH FIR matrices and they satisfy the condition given in Proposition 6. The FCT establishes a two-to-one mapping between SP FIR matrices and SPSH FIR matrices, as shown in Fig. 4.

Now we can find the inverse transform of the FCT and formulate an explicit formula for $\mathbf{U}(\boldsymbol{z})$. Suppose $\mathbf{H}^{\prime}(\boldsymbol{z})$ is a $2 \times 2 \mathrm{SPSH}$ FIR matrix with

$$
\mathbf{H}^{\prime}(\boldsymbol{z})=\left(\begin{array}{cc}
H_{00}^{\prime}(\boldsymbol{z}) & H_{01}^{\prime}(\boldsymbol{z}) \\
-H_{01}^{\prime}\left(\boldsymbol{z}^{-1}\right) & -H_{00}^{\prime}(\boldsymbol{z})
\end{array}\right)
$$

where $H_{00}^{\prime}(\boldsymbol{z})$ is an antisymmetric filter and $H_{01}^{\prime}(\boldsymbol{z})$ is an arbitrarily filter. By Theorem 2 , the CT of $\mathbf{H}(\boldsymbol{z})$ can be written as

$$
\mathbf{U}(\boldsymbol{z})=\operatorname{adj}\left(D(\boldsymbol{z}) \mathbf{I}+\mathbf{H}^{\prime}(\boldsymbol{z})\right)-\mathbf{I}=\left(\begin{array}{cc}
D(\boldsymbol{z})-H_{00}^{\prime}(\boldsymbol{z})-1 & -H_{01}^{\prime}(\boldsymbol{z}) \\
H_{01}^{\prime}\left(\boldsymbol{z}^{-1}\right) & D(\boldsymbol{z})+H_{00}^{\prime}(\boldsymbol{z})-1
\end{array}\right) .
$$

We summarize our results in the FCT domain in the following Theorem:

TheOREm 3. Suppose $\mathbf{H}^{\prime}(\boldsymbol{z})$ is a $2 \times 2$ SPSH FIR matrix. Then the inverse FIR-Cayley transform of $\mathbf{H}^{\prime}(\boldsymbol{z})$ exists if and only if $\sqrt{1-\operatorname{det}\left(\mathbf{H}^{\prime}(\boldsymbol{z})\right)}$ is FIR. If it exists, it is given by

$$
\mathbf{U}(\boldsymbol{z})= \pm \sqrt{1-\operatorname{det}\left(\mathbf{H}^{\prime}(\boldsymbol{z})\right)} \mathbf{I}-\mathbf{H}^{\prime}(\boldsymbol{z})
$$

Theorem 3 provides the characterization of $2 \times 2$ SP matrices in the Cayley domain. However, the condition for $\mathbf{H}^{\prime}(\boldsymbol{z})$ is complex. For implementation, we will design $D(\boldsymbol{z})$ and $\mathbf{H}^{\prime}(\boldsymbol{z})$ simultaneously in terms of $(20)$, and $D(\boldsymbol{z})$ is symmetric and $\mathbf{H}^{\prime}(\boldsymbol{z})$ is SPSH. Then we can compute the SP FIR matrix by $(21)$.

\subsection{Vanishing-Moment Condition and 2D Quincunx Orthogonal Filter Banks Design}

Quincunx sampling is density-2 sampling, leading thus again to the two-channel case. Of all MD sampling patterns, the quincunx one is the most common. However, since the sampling is nonseparable, it offers challenges.

In the context of wavelet design, the vanishing-moment condition plays an essential role. This condition requires the lowpass filter to have $L$-th order zero derivatives at $\boldsymbol{z}=[-1,-1]^{T}$. By Theorem 3 , the lowpass filer will be

$$
F\left(z_{1}, z_{2}\right)=z_{1}^{-1} H_{01}^{\prime}\left(z_{1}^{-1} z_{2}^{-1}, z_{1}^{-1} z_{2}\right)+D\left(z_{1} z_{2}, z_{1} z_{2}^{-1}\right)-H_{00}^{\prime}\left(z_{1} z_{2}, z_{1} z_{2}^{-1}\right)-1 .
$$

Therefore, the vanishing-moment condition imposes the values on the derivatives of $D(\boldsymbol{z}), H_{00}^{\prime}(\boldsymbol{z})$ and $H_{01}^{\prime}(\boldsymbol{z})$ at $\boldsymbol{z}=[-1,-1]^{T}$. If we parameterizes these 3 filters, then the vanishing-moment condition amounts to linear equations, which can be solved easily.

The design process is as follows: First, we parameterize the filters. Second, we compute the lowpass filter. Third, we impose the vanishing-moment condition on the lowpass filter. Finally, we solve the spectral factorization problem. The specific design procedure is given as follows.

1. Parameterize the symmetric FIR filter $D\left(z_{1}, z_{2}\right)$ and anti-symmetric FIR filter $H_{00}^{\prime}\left(z_{1}, z_{2}\right)$. 
2. Parameterize an FIR filter $H_{01}^{\prime}\left(z_{1}, z_{2}\right)$ of the same size.

3. Impose the $L$-th order vanishing moment on the lowpass filter given in (22):

$$
\left.\frac{\partial^{n} F\left(z_{1}, z_{2}\right)}{\partial^{i} z_{1} \partial^{n-i} z_{2}}\right|_{(-1,-1)}=0, \quad \text { for } \quad n=0,1, \ldots, L ; i=0,1, \ldots, n .
$$

It amounts to linear equations.

4. Solve the spectral factorization equation by comparing the coefficients of $\boldsymbol{z}$ :

$$
D^{2}\left(z_{1}, z_{2}\right)-2 D\left(z_{1}, z_{2}\right)+H_{01}^{\prime}\left(z_{1}, z_{2}\right) H_{01}^{\prime}\left(z_{1}^{-1}, z_{2}^{-1}\right)-H_{00}^{\prime 2}\left(z_{1}, z_{2}\right)=0 .
$$

It amounts to quadratic equations.

5. Compute $\mathbf{U}\left(z_{1}, z_{2}\right)$ by $(21)$.

\section{ORTHOGONAL IIR FILTER BANKS}

As we said, designing orthogonal IIR filter banks is more complex than designing FIR orthogonal ones, although it has greater freedom. For implementation purposes, we only consider recursive filters, $z$-transforms of which are rational functions. It is straightforward to design an orthogonal filter bank in the Cayley domain via a PSH matrix, and then map it back to a paraunitary polyphase matrix. Therefore, the range of paraunitary matrices in the Cayley domain is the set of all PSH matrices. In this section, unless explicitly stated otherwise, assume that $\mathbf{U}(\boldsymbol{z})$ is an $N \times N$ paraunitary IIR matrix and $\mathbf{H}(\boldsymbol{z})$ is its CT.

THEOREM 4. The range of paraunitary IIR matrices in the Cayley domain is a linear space.

Take the two-channel case as an example. For the multiple-channel case, we have similar results. Let $\mathbf{H}(\boldsymbol{z})$ be a PSH matrix

$$
\mathbf{H}(\boldsymbol{z})=\left(\begin{array}{cc}
H_{00}(\boldsymbol{z}) & H_{01}(\boldsymbol{z}) \\
H_{10}(\boldsymbol{z}) & H_{11}(\boldsymbol{z})
\end{array}\right) .
$$

Then the PSH condition implies

$$
H_{00}\left(\boldsymbol{z}^{-1}\right)=-H_{00}(\boldsymbol{z}), \quad H_{11}\left(\boldsymbol{z}^{-1}\right)=-H_{11}(\boldsymbol{z}), \quad \text { and } \quad H_{10}\left(\boldsymbol{z}^{-1}\right)=-H_{01}(\boldsymbol{z}) .
$$

We can select nondiagonal entries arbitrarily (under the constraint $H_{10}\left(\boldsymbol{z}^{-1}\right)=-H_{01}(\boldsymbol{z})$ ). For the diagonal entries, we just need to find anti-symmetric IIR filters. Then the problem of designing $\mathbf{H}(\boldsymbol{z})$ converts to that of designing anti-symmetric IIR filters.

Proposition 7. Suppose $w(\boldsymbol{z})$ be an IIR filter with a rational function: $W(\boldsymbol{z})=a(\boldsymbol{z}) / b(\boldsymbol{z})$, where $a(\boldsymbol{z})$ and $b(\boldsymbol{z})$ are coprime polynomials. If $w\left(\boldsymbol{z}^{-1}\right)=-w(\boldsymbol{z})$, then

$$
a\left(\boldsymbol{z}^{-1}\right)=c \boldsymbol{z}^{\boldsymbol{k}} a(\boldsymbol{z}) \quad \text { and } \quad b\left(\boldsymbol{z}^{-1}\right)=-c \boldsymbol{z}^{\boldsymbol{k}} b(\boldsymbol{z}),
$$

where $\boldsymbol{k}$ is an arbitrary integer.

\subsection{Vanishing-Moment Condition and Simplifications}

The CT has a simple form for the design of orthogonal IIR filter banks. It avoids the problem of spectral factorization, which is difficult (if possible) in the MD case. As in the orthogonal FIR case, the vanishingmoment condition plays an essential role in the context of wavelet design. This condition requires the lowpass filter to have specific order zero derivatives at $\boldsymbol{z}=[-1,-1,-1, \ldots,-1]^{T}$. However, it is difficult to impose the vanishing-moment condition on MD orthogonal IIR filters because it involves rational functions in the design. First, many parameters are required. For each filter in $\mathbf{H}(\boldsymbol{z})$, not only the numerator but also the denominator requires parameters. Second, to impose the vanishing-moment condition on the IIR filter, we need to solve nonlinear equations with respect to those parameters (as opposed to the linear equations in the FIR case). Therefore, we need to simplify the design of orthogonal IIR filter banks. 
As mentioned in Section 3, SP matrices are simplified paraunitary matrices and each paraunitary matrix can be "normalized" as a paraunitary matrix with only a phase change in one highpass filter. This does not affect the vanishing-moment condition. Therefore, we can simplify our design by SP matrices. In this subsection, we only consider the two-channel case.

In the polyphase domain, the characterization of MD orthogonal IIR filter banks is SP matrices. The characterization of MD SP matrices in the Cayley domain is SPSH matrices. By Proposition 4, a $2 \times 2$ SPSH matrix, $\mathbf{H}(\boldsymbol{z})$, can be formulated as

$$
\mathbf{H}(\boldsymbol{z})=\left(\begin{array}{cc}
f(\boldsymbol{z}) & g(\boldsymbol{z}) \\
-g\left(\boldsymbol{z}^{-1}\right) & -f(\boldsymbol{z})
\end{array}\right)
$$

where $f(\boldsymbol{z})$ is an anti-symmetric filter, and $g(\boldsymbol{z})$ is an arbitrary filter.

The set of SPSH matrices is a linear space. Obviously, every SPSH matrix can be represented as the sum of a diagonal matrix and an anti-diagonal matrix, i.e.,

$$
\mathbf{H}(\boldsymbol{z})=\left(\begin{array}{cc}
f(\boldsymbol{z}) & 0 \\
0 & -f(\boldsymbol{z})
\end{array}\right)+\left(\begin{array}{cc}
0 & g(\boldsymbol{z}) \\
-g\left(\boldsymbol{z}^{-1}\right) & 0
\end{array}\right) .
$$

Note they are independent and both of them are SPSH matrices with simple structures. The first one is called the diagonal SPSH (DSPSH) matrix and the second one is called anti-diagonal SPSH (ASPSH) matrix. Correspondingly, their CTs also have simple structures. The CT of a DSPSH matrix is

$$
\mathbf{U}(\boldsymbol{z})=\left(\begin{array}{cc}
\frac{1-f(\boldsymbol{z})}{1+f(\boldsymbol{z})} & 0 \\
0 & \frac{1+f(\boldsymbol{z})}{1-f(\boldsymbol{z})}
\end{array}\right) .
$$

The CT of ASPSH matrix is

$$
\mathbf{U}(\boldsymbol{z})=\frac{1}{1+g(\boldsymbol{z}) g\left(\boldsymbol{z}^{-1}\right)}\left(\begin{array}{cc}
1-g(\boldsymbol{z}) g\left(\boldsymbol{z}^{-1}\right) & -2 g(\boldsymbol{z}) \\
2 g\left(\boldsymbol{z}^{-1}\right) & 1-g(\boldsymbol{z}) g\left(\boldsymbol{z}^{-1}\right)
\end{array}\right) .
$$

In the sequel, we only consider $1 \mathrm{D}$ case. For a $1 \mathrm{D}$ orthogonal IIR filter of the $N$-th order vanishing-moment, it can be factored as $\left(1+z^{-1}\right)^{N} R(z) / D(z)$, where $R(z)$ and $D(z)$ are FIR, and $D(-1) \neq 0$. Moreover, if $R(z) \equiv 1$ and $D(z)$ has the lowest order, then it is called a Butterworth filter. Otherwise, it is an intermediate filter.

First consider DSPSH matrices. From (25), the lowpass filer is $U_{0}(z)=\left(1+f\left(z^{2}\right)\right)^{-1}\left(1-f\left(z^{2}\right)\right)$. Impose the $N$-th vanishing-moment condition, then $1-f\left(z^{2}\right)=\left(1+z^{-1}\right)^{N} R(z)$. Note $f\left(z^{-1}\right)=-f(z)$, then $1+f\left(z^{2}\right)=$ $1-f\left(z^{-2}\right)=(1+z)^{N} R\left(z^{-1}\right)$ and $U_{0}(z)=z^{-N} R(z) / R\left(z^{-1}\right)$. Hence, the corresponding low-pass filter cannot have vanishing moments.

Now consider ASPSH matrices. To simplify the problem, we make one assumption: $g(z) g\left(z^{-1}\right)=c^{2}$, where $c$ is a nonzero constant. Even with this simplification, we still have many design results. The following proposition gives the characterization of filters under such assumption.

Proposition 8. Suppose $g(z)$ is a rational filter with the form $g\left(z^{2}\right)=p(z) / q(z)$, where $p(z)$ and $q(z)$ are coprime FIR filters. Then $g(z) g\left(z^{-1}\right)$ equals to a constant if and only if $p(z)=c z^{k} q\left(z^{-1}\right)$, where $c$ is a nonzero constant, and $k$ is an integer.

By this simplification, the low-pass filter can be represented as $U_{0}(z)=\left(1+c^{2}\right)^{-1}\left(1-c^{2}-2 z^{-1} g\left(z^{2}\right)\right)$. By Proposition 8, it can be further formulated as

$$
U_{0}(z)=\frac{\left(1-c^{2}\right) q(z)-2 c z^{k-1} q\left(z^{-1}\right)}{\left(1+c^{2}\right) q(z)} .
$$

Now consider the $N$-th vanishing-moment condition, and then $\left(1-c^{2}\right) q(z)-2 c z^{k-1} q\left(z^{-1}\right)=\left(1+z^{-1}\right)^{N} R(z)$, where both $q(z)$ and $R(z)$ are FIR and $q(-1) \neq 0, R(-1) \neq 0$. Solutions are given in the following Theorem:

Proposition 9. A low-pass filter $U_{0}(z)$ generated by an ASPSH matrix is an orthogonal filter with the $N$-th order vanishing-moment if and only if one of the following conditions holds: 
1. $\left(1-c^{2}\right)^{2} \neq 4 c^{2}$, and

$$
q(z)=\frac{1}{\left(1-c^{2}\right)^{2}-4 c^{2}}\left(2 c z^{k-1}(1+z)^{N} R\left(z^{-1}\right)+\left(1-c^{2}\right)\left(1+z^{-1}\right)^{N} R(z)\right) .
$$

2. $c=1 \pm \sqrt{2}, R\left(z^{-1}\right)=z^{1-k-N} R(z)$, and

$$
q(z)=s(z)-\frac{1}{4 c}\left(1+z^{-1}\right)^{N} R(z)
$$

where $s(z)$ is an arbitrary nonzero antisymmetric filter such that $s\left(z^{-1}\right)=-z^{1-k} s(z)$.

3. $c=-1 \pm \sqrt{2}, R\left(z^{-1}\right)=-z^{1-k-N} R(z)$, and

$$
q(z)=s(z)+\frac{1}{4 c}\left(1+z^{-1}\right)^{N} R(z)
$$

where $s(z)$ is an arbitrary nonzero symmetric filter such that $s\left(z^{-1}\right)=z^{1-k} s(z)$.

If we let $R(z)$ be 1 in Proposition 9, then we generate Butterworth filters. Herley and Vetterli also got the close solutions for 1D $N$-th order Butterworth filters in [13].

\section{CONCLUSION}

We presented the complete characterization of both paraunitary FIR matrices and paraunitary IIR matrices in the Cayley domain and proposed efficient design methods for MD orthogonal filter banks and corresponding methods to impose the vanishing-moment condition.

\section{REFERENCES}

1. T. Chen and P. P. Vaidyanathan, "Multidimensional multirate filters and filter banks derived from one-dimensional filters," IEEE Trans. Signal Proc., vol. 41, no. 5, pp. 1749-1765, May 1993.

2. — - "Recent developments in multidimensional multirate systems," IEEE Trans. on CSVT, vol. 3, no. 2, pp. 116-137, April 1993.

3. R. H. Bamberger and M. J. T. Smith, "A filter bank for the directional decomposition of images: Theory and design," IEEE Trans. Signal Proc., vol. 40, no. 4, pp. 882-893, April 1992.

4. K. Gröchenig and W. R. Madych, "Multiresolution analysis, Haar bases and self-similar tilings of $R^{n}$," IEEE Transactions on Information Theory, Special Issue on Wavelet Transforms and Multiresolution Signal Analysis, vol. 38, no. 2, pp. 556-568, March 1992.

5. G. Karlsson and M. Vetterli, "Theory of two - dimensional multirate filter banks," IEEE Trans. Acoust., Speech, and Signal Proc., vol. 38, no. 6, pp. 925-937, June 1990.

6. M. Vetterli and J. Kovačević, Wavelets and Subband Coding. Englewood Cliffs, NJ: Prentice-Hall, 1995.

7. E. Viscito and J. P. Allebach, "The analysis and design of multidimensional FIR perfect reconstruction filter banks for arbitrary sampling lattices," IEEE Trans. Circ. and Syst., vol. 38, no. 1, pp. 29-42, January 1991.

8. D. B. H. Tay and N. G. Kingsbury, "Flexible design of multidimensional perfect reconstruction FIR 2-band filters using transformations of variables," IEEE Trans. Image Proc., vol. 2, no. 4, pp. 466-480, October 1993.

9. J. Kovačević and M. Vetterli, "Fco sampling of digital video using perfect reconstruction filter banks," IEEE Trans. Image Proc., vol. 2, no. 1, pp. 118-122, January 1993.

10. I. Daubechies, "Orthonormal bases of compactly supported wavelets," Commun. on Pure and Appl. Math., vol. 41, pp. 909-996, November 1988.

11. M. J. T. Smith and T. P. Barnwell III, "Exact reconstruction for tree-structured subband coders," IEEE Trans. Acoust., Speech, and Signal Proc., vol. 34, no. 3, pp. 431-441, June 1986.

12. P. P. Vaidyanathan, Multirate Systems and Filter Banks. Englewood Cliffs, NJ: Prentice-Hall, 1993.

13. C. Herley and M. Vetterli, "Wavelets and recursive filter banks," IEEE Trans. Signal Proc., vol. 41, no. 8, pp. 2536-2556, August 1993.

14. B. Hassibi and B. M. Hochwald, "Cayley differential unitary space-time codes," IEEE Trans. Info. Theory, vol. 48, no. 6, pp. 1485-1503, June 2002.

15. J. Zhou, M. N. Do, and J. Kovačcević, "New design of orthogoanl filter banks and wavelets using the cayley transform," IEEE Trans. Signal Proc., 2003, to be submitted.

16. A. V. Oppenheim, R. W. Schafer, and J. R. Buck, Discrete-Time Signal Processing. Englewood Cliffs, NJ: PrenticeHall, 1999. 